\title{
Combined Duval Pentagons: A Simplified Approach
}

\author{
Luiz Cheim $^{1, *}$, Michel Duval ${ }^{2}$ and Saad Haider ${ }^{1}$ \\ 1 Transformer Technology Center, ABB Power Grids, St. Louis, MO 63127, USA; saad.haider@us.abb.com \\ 2 Institut de Recherche d'Hydro-Québec (IREQ), Varennes, QC J3X 1S1, Canada; Duval.Michel@ireq.ca \\ * Correspondence: luiz.v.cheim@us.abb.com; Tel.: +1-314-452-1445
}

Received: 10 April 2020; Accepted: 2 June 2020; Published: 3 June 2020

\begin{abstract}
The paper describes a newly proposed combination of the two existing Duval Pentagons method utilized for the identification of mineral oil-insulated transformers. The aim of the combination is to facilitate automatic fault identification through computer programs, and at the same time, apply the full capability of both original Pentagons, now reduced to a single geometry. The thorough classification of a given fault (say, of the electrical or thermal kind), employing individual Pentagons 1 and 2, as originally defined, involves a complex geometrical problem that requires the build-up of a convoluted geometry (a regular Pentagon whose axes represent each of five possible combustible gases) to be constructed using computer language code and programming, followed by the logical localization of the geometrical centroid of an irregular pentagon, formed by the partial contribution of individual combustibles, inside two similar structures (Pentagons 1 and 2) that, nonetheless, have different classification zones and boundaries, as more thoroughly explained and exemplified in the main body of this article. The proposed combined approach results in a lower number of total fault zones (10 in the combined Pentagons against 14 when considering Pentagons 1 and 2 separately, although zones PD, S, D1 and D2 are common to both Pentagons 1 and 2), and therefore eliminates the need to solve for two separate Pentagons.
\end{abstract}

Keywords: transformer; DGA; Duval pentagons; fault classification; combined pentagons

\section{Introduction}

Dissolved gas analysis (DGA) in oil insulated transformers has been widely used to detect incipient faults, typically associated to the level of certain combustible gases in the oil, as well as their historical trends. There are several methods available for the interpretation of DGA from laboratory results, such as those recommended in the IEC 60599-2015 09 [1] and IEEE C57.104-2019 [2]. The methods indicated in those Standards are fundamentally based on the computation of certain combustible gas ratios for which there has been historical association to the development of actual faults in transformers, validated by the collection of large datasets from transformers in service, for which the corresponding DGA data was recorded and compared to the actual faults identified after internal inspection, frequently carried out by experts and maintenance personnel after transformer failure, and sometimes just before major failures take place.

The gas ratios calculated as indicated in those Standards can be easily converted into graphical visualization using two-dimension and even three-dimension charts, as in the IEC method. Since there is always an inherent error in the measurement of the quantities of dissolved gases in the oil, as indicated in the IEC document, there will certainly be uncertainty also propagated when the proposed gas ratios are calculated. These uncertainties may affect the interpretation of DGA, particularly when graphical techniques are employed. The accuracy aspect of DGA with respect to the use of the proposed Duval Pentagons will be discussed below in Section 6.

When the methods above are utilized to detect incipient faults in transformers, investigators are commonly concerned with some special types of faults, in particular, the so-called high-energy 
faults of very high temperature or arcing, due to the fact that such faults may quickly lead to major equipment failures, and also with the involvement or not involvement of cellulose (paper insulation) in the developing fault. The indication of paper involvement in the fault may lead researchers to the conclusion that the fault is developing in the transformer active part, or at least in a region covered by paper insulation such as leads and tap connections.

The diagnostic of paper involvement and the eventual carbonization of the solid insulation can be reinforced by the careful use of $\mathrm{CO}$ (carbon monoxide) and $\mathrm{CO}_{2}$ (carbon dioxide), plus additional aging markers, such as Furans. Furans constitute a family of chemical compounds that are found dissolved in the transformer-insulating oil but that cannot be produced by the oil itself. It is known to be produced by the degradation of cellulose, and therefore it is thought to be a powerful additional tool to be utilized in the analysis of paper degradation. References $[3,4]$ provide a thorough review of the state-of-the-art application of Furans, as well as the difficulties associated to the quantitative correlation between Furans and the actual degree of polymerization of the paper (DP).

The graphical DGA method employed in the present article is based on the original Duval Pentagons [5] that are also part of the recently revised IEEE C57.104 [2].

\section{Original Duval Pentagon Method}

Before moving into the explanation of the combined approach it is necessary to clarify a few points about the use of the Pentagon method and briefly describe the utilization of each Pentagon. The DGA methods indicated in the Introduction typically use ratios of the five main hydrocarbon gases found dissolved in the oil, namely $\mathrm{H}_{2}$ (hydrogen), $\mathrm{CH}_{4}$ (methane), $\mathrm{C}_{2} \mathrm{H}_{6}$ (ethane), $\mathrm{C}_{2} \mathrm{H}_{4}$ (ethylene), and $\mathrm{C}_{2} \mathrm{H}_{2}$ (acetylene).

As explained in [5], the relative percentages of the five main hydrocarbon gases are calculated (ppm values) and the relative contribution of each gas is found by dividing the amount of that gas by the sum of all five components. For example, to find the hydrogen contribution in percent calculate $\mathrm{H}_{2}(\%)=\left(\mathrm{H}_{2}\right.$ in ppm $) /\left(\mathrm{H}_{2}+\mathrm{CH}_{4}+\mathrm{C}_{2} \mathrm{H}_{6}+\mathrm{C}_{2} \mathrm{H}_{4}+\mathrm{C}_{2} \mathrm{H}_{2}\right.$, all in ppm $)$.

Once the relative contributions are calculated they are plotted on a regular Pentagon whose axes are formed by each of these gases, as illustrated in Figure 1a. As shown, each summit of the pentagon corresponds to one gas, e.g., $\mathrm{H}_{2}, \mathrm{C}_{2} \mathrm{H}_{6}$, etc. Following the definition, each axis contains up to $100 \%$ contribution of each gas but in practice this is never the case, as explained below.

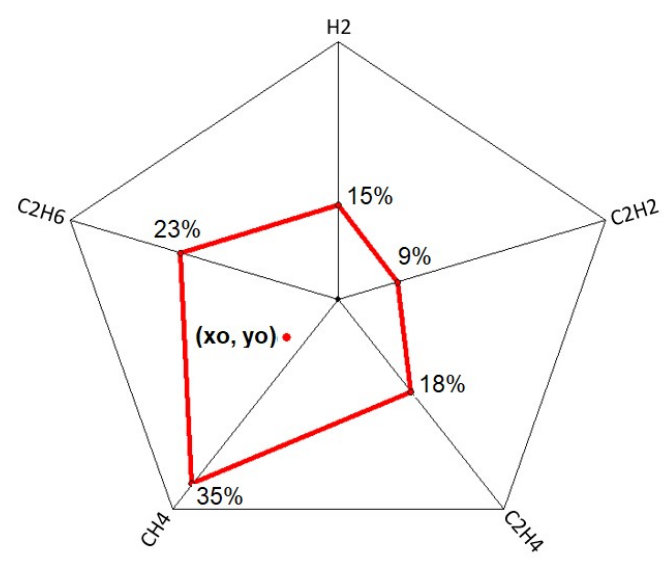

(a)

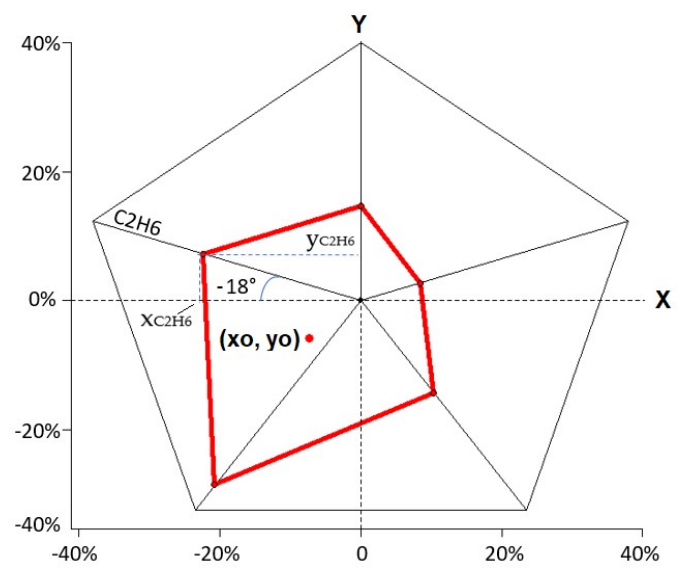

(b)

Figure 1. Original Duval Pentagon (a) Regular pentagon formed by the five axes, corresponding to the relative contribution, in percent, of each of the five hydrocarbons $\left(\mathrm{H}_{2}, \mathrm{CH}_{4}, \mathrm{C}_{2} \mathrm{H}_{2}, \mathrm{C}_{2} \mathrm{H}_{4}, \mathrm{C}_{2} \mathrm{H}_{6}\right)$ (b) determination of the coordinates of each combustible gas, projected onto a system of Cartesian coordinates. The redlined polygon shows the case for the gas $\mathrm{C}_{2} \mathrm{H}_{6}=23 \%$, as in the example given above. 
For a given DGA sample, the relative percentage of $\mathrm{H}_{2}$ is plotted on the axis between the pentagon center $\left(0 \% \mathrm{H}_{2}\right)$ and the pentagon summit for $\mathrm{H}_{2}$ (vertical axis). The same is done for each of the other four gases, as illustrated in Figure $1 \mathrm{a}$, for a gas sample showing $\mathrm{H}_{2}=50 \mathrm{ppm}, \mathrm{CH}_{4}=120 \mathrm{ppm}$, $\mathrm{C}_{2} \mathrm{H}_{2}=30 \mathrm{ppm}, \mathrm{C}_{2} \mathrm{H}_{4}=60 \mathrm{ppm}$, and $\mathrm{C}_{2} \mathrm{H}_{6}=80 \mathrm{ppm}$, yielding the following approximate relative percentage contribution of each gas: $\mathrm{H}_{2}=15 \%, \mathrm{CH}_{4}=35 \%, \mathrm{C}_{2} \mathrm{H}_{2}=9 \%, \mathrm{C}_{2} \mathrm{H}_{4}=18 \%$ and $\mathrm{C}_{2} \mathrm{H}_{6}=23 \%$.

Each of these relative contributions is plotted in the corresponding gas axis, as illustrated in Figure $1 \mathrm{a}$, then forming the generic irregular polygon of five sides (outlined in red). The ultimate point of interest for the diagnostic and final classification of the Dissolved Gas Analysis, using the Pentagon method, is the geometrical centroid (xo; yo), the red dot in the center of the irregular polygon, as illustrated below in Figure 1.

In order to mathematically find the centroid of the irregular polygon, one needs to know the coordinates $\mathrm{x}, \mathrm{y}$ for each individual gas. For the example illustrated in Figure $1 \mathrm{a}$, take the axis for the gas $\mathrm{C}_{2} \mathrm{H}_{6}$, whose angle $\alpha$ between the $\mathrm{C}_{2} \mathrm{H}_{6}$ axis and the horizontal $\mathrm{x}$ axis in Figure $1 \mathrm{~b}$ is $180^{\circ}-18^{\circ}$ degrees. This leads to the following $\mathrm{x}$; $\mathrm{y}$ coordinates for the gas $\mathrm{C}_{2} \mathrm{H}_{6}$ inside the Pentagon:

$$
\begin{aligned}
& \mathrm{x}_{\mathrm{C}_{2} \mathrm{H}_{6}}=23 \% \times \cos (\alpha)=23 \times \cos \left(180^{\circ}-18^{\circ}\right)=23 \times-0.95=-21.85 \\
& \mathrm{y}_{\mathrm{C}_{2} \mathrm{H}_{6}}=23 \% \times \sin (\alpha)=23 \times \sin \left(180^{\circ}-18^{\circ}\right)=23 \times 0.31=7.13
\end{aligned}
$$

The same procedure is applied to the other remaining four gases and their respective coordinates, using the angles between each gas axis and the reference coordinate system. The centroid of the irregular polygon drawn from the five points is then found mathematically by the application of Equations (1)-(3) below.

$$
\begin{gathered}
C_{x}=\frac{1}{6 A} \sum_{i-0}^{n-1}\left(x_{i}+x_{i+1}\right)\left(x_{i} y_{i+1}-x_{i+1} y_{i}\right) \\
C_{y}=\frac{1}{6 A} \sum_{i}\left(y_{i}+y_{i+1}\right)\left(x_{i} y_{i+1}-x_{i+1} y_{i}\right) \\
A=\frac{1}{2} \sum_{i-0}^{n-1}\left(x_{i} y_{i+1}-x_{i+1} y_{i}\right)
\end{gathered}
$$

where $C_{x}$ and $C_{y}$ are the Cartesian coordinates of the centroid formed by the irregular polygon, and $A$ is the area of the same polygon.

In the example shown in Figure 1, the coordinates of the centroid are xo $=-7.35$, and yo $=-5.79$. It should be noted, as pointed out in [5], that the center of an irregular polygon may also be calculated as its center of mass or the average of the $x_{i}$ and $y_{i}$ coordinates of the polygon. The centroid, as calculated above, is the preferred method as originally presented by Michel Duval in [5].

Another important aspect of the original Pentagon is the sequence of gases that follows the increasing level of energy required to produce those very gases that appear in the summits of the Pentagon, as in $\mathrm{H}_{2} \rightarrow \mathrm{C}_{2} \mathrm{H}_{6} \rightarrow \mathrm{CH}_{4} \rightarrow \mathrm{C}_{2} \mathrm{H}_{4} \rightarrow \mathrm{C}_{2} \mathrm{H}_{2}$, always counterclockwise, as illustrated in Figure 1. This order has demonstrated to be the best fit in the process of fault identification in transformers using the Pentagon method.

The reader may be surprised by the fact that the maximum level that appears in the summits of the Pentagon is $40 \%$. This level for $\mathrm{H}_{2}$ in Figure $1 \mathrm{~b}$ can be explained by the fact that even when one gas (say, $\mathrm{H}_{2}$ ) has a contribution of $100 \%$, hence all others must add up to zero, the centroid itself (and not the contribution of $\mathrm{H}_{2}$ ) will never fall on a level that is superior to $40 \%$.

Using Equations (1)-(3), it is possible to simulate the centroid of the irregular polygon given some extreme conditions, for example: $\mathrm{H}_{2}=100 \mathrm{ppm}$, all other gases $=0$; centroid $(100,0,0,0,0)=(0 ; 33.3)$; $\mathrm{CH}_{4}=100 \mathrm{ppm}$, all other gases $=0$; $\operatorname{centroid}(0,100,0,0)=(-19.5 ;-26.9) ; \mathrm{C}_{2} \mathrm{H}_{6}=100$, all other gases = 0 ; centroid $(0,0,0,0,100)=(-31.6 ; 10.3)$.

Therefore, whatever the gas levels in ppm, when their relative contributions are taken into account, as explained above, the axes that correspond to each combustible may be limited to $40 \%$ as shown. The summits of the original Pentagon are consequently defined by the following Cartesian coordinates: 


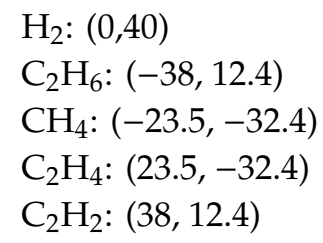

\section{Fault Zones in the Duval Pentagon 1}

The procedure above explains the formation of the original Duval Pentagon and the location of the centroid, for a particular DGA sample, of an irregular pentagon, when the contribution of the five hydrocarbons are represented inside that pentagon by their relative contributions and their respective geometrical centroid. In order to define the so-called fault zones in the Pentagon, this geometrical procedure was applied to a large number of actual DGA samples, from which there was visual inspection inside the transformers in order to identify the eventual electrical or thermal fault, or even a combination of both that might be occurring, since those DGA cases came from suspicious transformers, or transformers that investigators believed to be developing some issue, given their gas level, their gas formation rate, or both. The location of those DGA samples inside the Pentagon helped to outline the six main zones typically associated to electrical or thermal fault, as below:

- PD: corona partial discharges,

- D1: low energy electrical discharges,

- D2: high energy electrical discharges,

- $\quad$ T3: high temperature phenomena (thermal faults $>700^{\circ} \mathrm{C}$ ),

- T2: high temperature phenomena (thermal faults of 300 to $700^{\circ} \mathrm{C}$ ), and

- T1: overheating (thermal faults $<300{ }^{\circ} \mathrm{C}$ ).

In addition, it was also possible to identify stray gassing mineral oils, or oils that might be producing combustible gases under thermal stresses, but those gases would not necessarily be associated to electrical or thermal faults in the active part or main conductors of the transformer. Those are the so-called "S" fault zones. Figure $2 \mathrm{a}, \mathrm{b}$ illustrate the original Pentagon, as in Figure $1 \mathrm{a}, \mathrm{b}$, but now with the outlined fault zones based on the actual DGA samples, as explained above. The Cartesian coordinates that define all zones in Pentagon 1 are shown in Figure 3. The axes representing each combustible gas in Figure 1a were removed in Figure $1 \mathrm{~b}$ to avoid confusion with the fault zones.

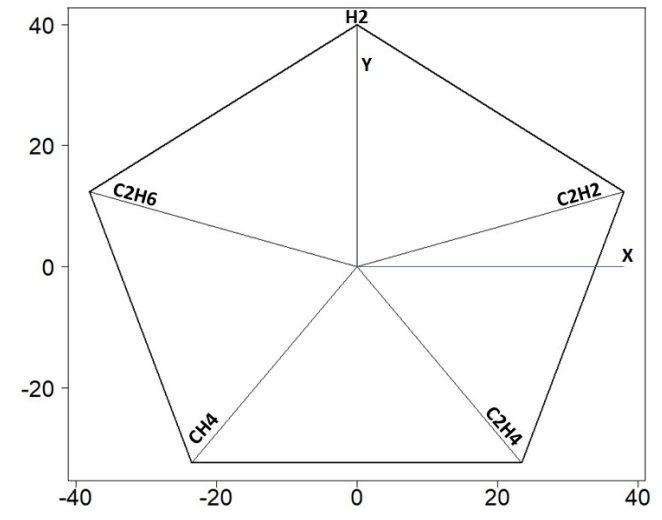

(a)

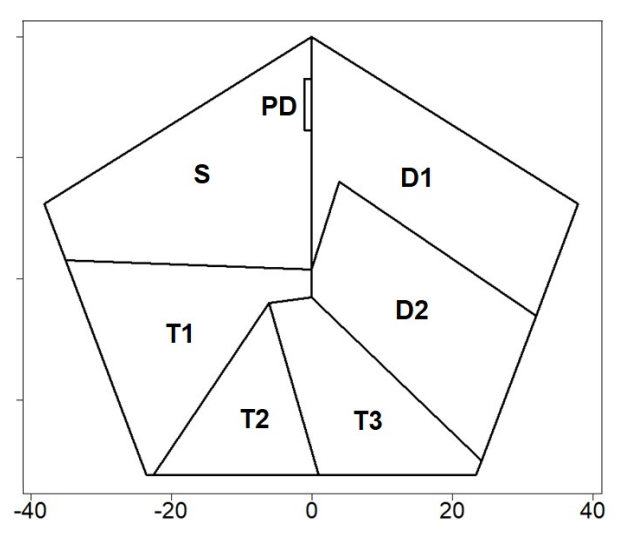

(b)

Figure 2. Duval Pentagon method (a) original regular Pentagon, formed by the five combustibles (b) Duval Pentagon 1, showing the fault zones as described above. 


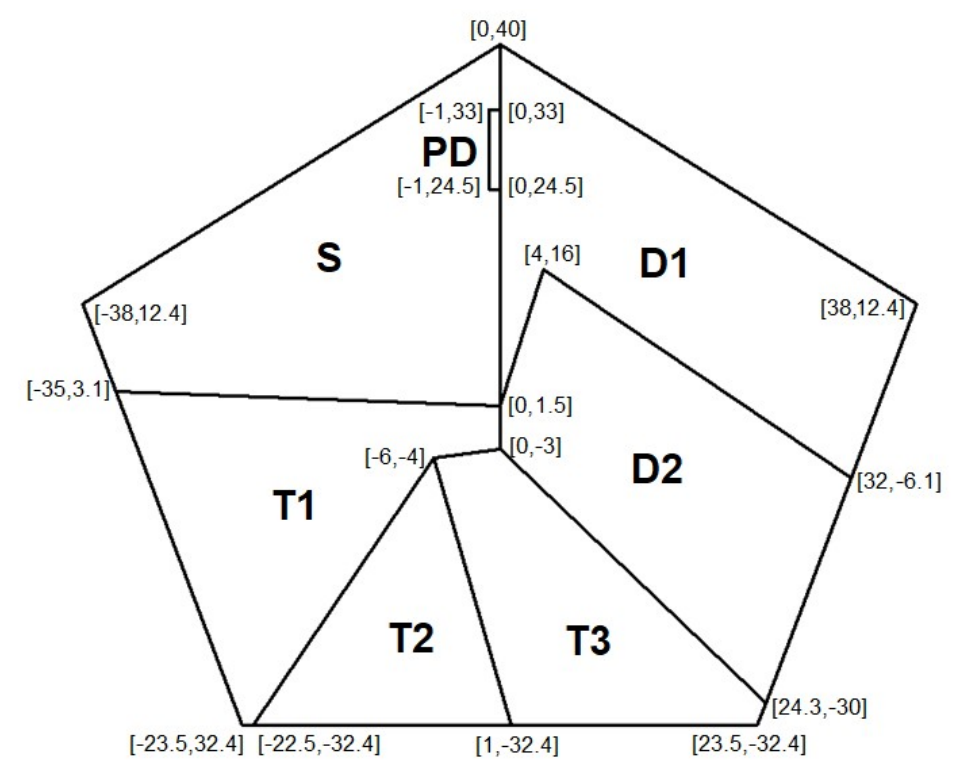

Figure 3. Coordinates of all seven fault zones in Pentagon 1 (PD, S, T1, T2, T3, D1 and D2).

\section{Fault Zones in Duval Pentagon 2}

When a transformer is exhibiting some sort of thermal fault (T1, T2 or T3 in Pentagon 1), it is highly desirable to identify the presence of paper carbonization involved in the fault, since this may be one of the most important factors that may lead users and experts alike to the decision of removing the transformer from operation to proceed with further investigation or even with a more drastic decision as to repair or replace the unit, depending on the assessment of the level of severity of the problem. This advanced diagnostic can be achieved by the better resolution given by the application of Pentagon 2, illustrated in Figure 4, in which the thermal faults are now classified into " $\mathrm{C}$ " for carbonization, " $\mathrm{O}$ " for overheating and "T3- $\mathrm{H}$ " which are high temperature faults occurring in oil only. Notice that the fault zones S, PD, D1 and D2 in Pentagon 1 remain unaltered in Pentagon 2, since those do not represent thermal issues but instead, they are associated to electrical faults or stray gassing problems. Figure 4 also shows the coordinates of the new fault zones.

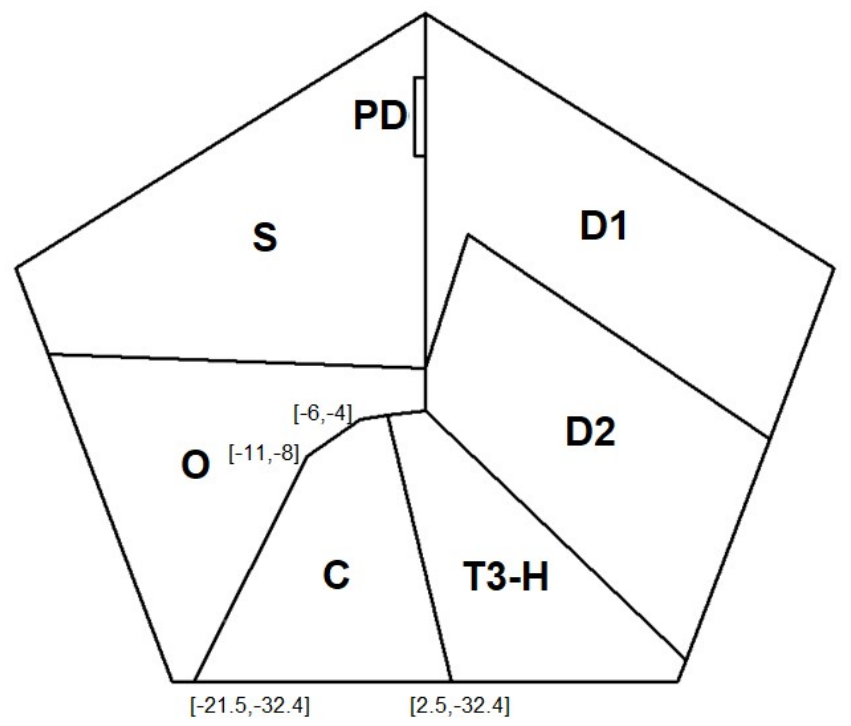

Figure 4. Duval Pentagon 2 with the new coordinates of fault zones O, C and T3-H. All other coordinates remain the same as in Pentagon 1, Figure 3. 


\section{The Combined Duval Pentagons}

As mentioned above, Pentagons 1 and 2 share the same geometry for zones PD, S, D1 and D2. The only significant difference between the two Pentagons occurs in the southern hemisphere involving thermal faults of type T1, T2 and T3 (Pentagon 1) that may be classified as O, C or T3-H in Pentagon 2. A visual inspection of both Figure $5 a, b$ below clearly indicate the similarities in the non-thermal zones and the significant changes in the thermal zones between Pentagons 1 and 2. It is possible to visually identify zone $C$ in Pentagon 2 covering almost the whole of T2 in Pentagon 1, part of T1 and T3, also in Pentagon 1. We can certainly see, for example, that T3-H (high temperature fault in oil only) in Pentagon 2 is a bit narrower than T3 in Pentagon 1, meaning that not all T3 occurs in oil only. Some T3, therefore, may involve carbonization of the insulation, zone C. The fact that T2 in Pentagon 1 is almost thoroughly encompassed by $\mathrm{C}$ in Pentagon 2, means that most thermal faults in the $300-500{ }^{\circ} \mathrm{C}$ range involve paper carbonization.

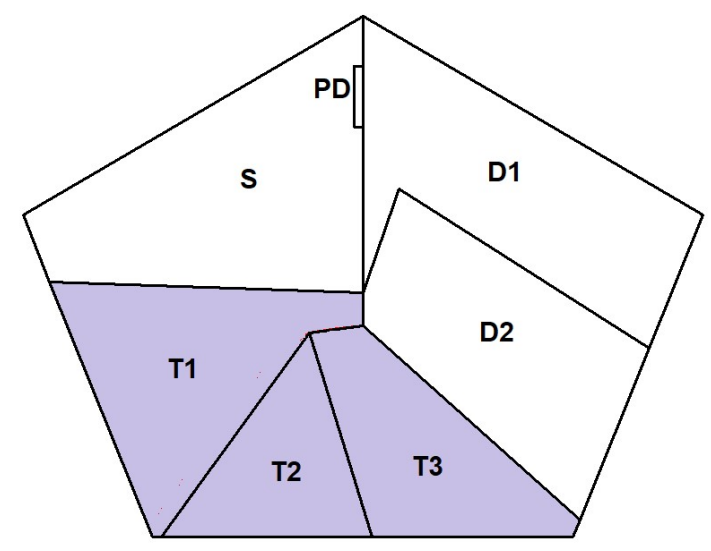

(a)

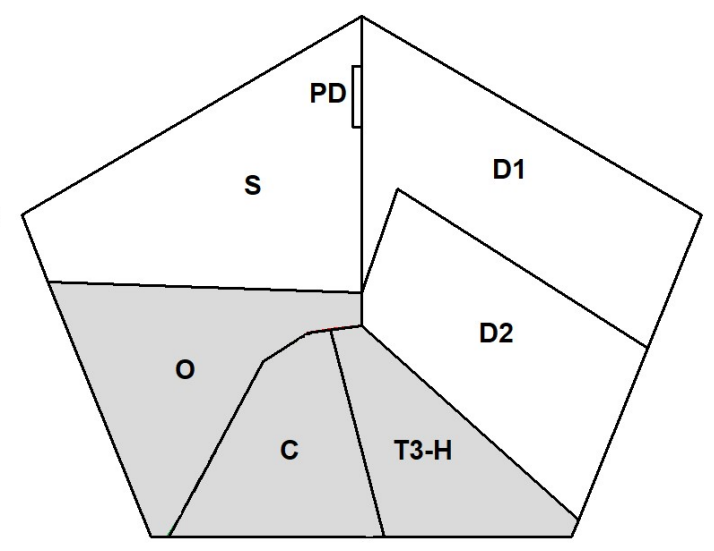

(b)

Figure 5. Shaded areas Illustrating differences between (a) thermal zones T1, T2 and T3. in Pentagon 1 (b) thermal zones O, C and T3-H in Pentagon 2.

Finally, most of T1 in Pentagon 1 are of type O in Pentagon 2, meaning that most thermal faults $<300{ }^{\circ} \mathrm{C}$ are classified as simple overheating. All these can be readily seen if we superimpose the geometries and coordinates of Pentagons 1 and 2.

Figure 6 shows the combined Pentagons, by superposition of Pentagons 1 and 2, with clear identification of all zones, including intersecting zones between Pentagons 1 and 2 . As can be readily seen from Figure $6 a, b$, there are four intersecting zones between zone " $C$ " in Pentagon 2 and zones T1, T2 and T3 in Pentagon 1. Three of those zones indicate possible paper carbonization associated to either T1, T2 or T3. One tiny zone on the bottom left indicates thermal issues of T2 type that would fall outside the "C" zone in Pentagon 2, therefore not involving carbonization.

It is then possible to utilize the two combined Pentagons, as described above, and define new names for the four intersecting zones. The new names are just simple combinations of the existing names in Pentagons 1 and 2, seen simultaneously in the newly combined geometry illustrated in Figures 7 and 8 below. Figure 9 shows the final geometry of the Combined Pentagons. 


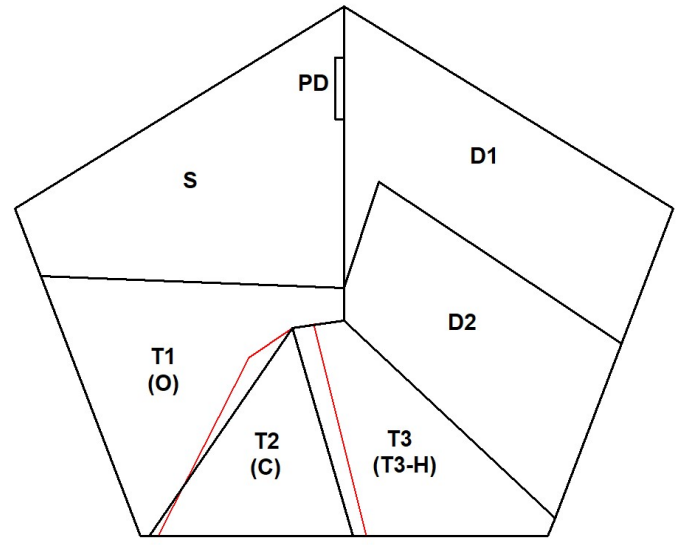

(a)

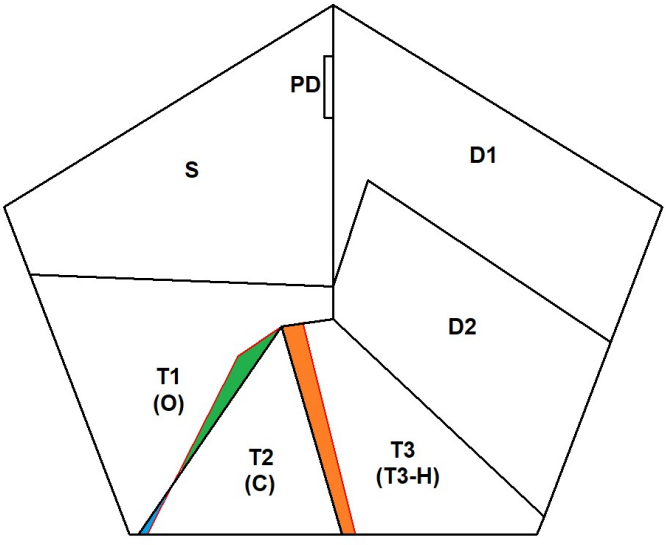

(b)

Figure 6. Superimposing the original Pentagons 1 and 2 (a) showing the original zones in both Pentagons 1 and 2 and redlining zone $C$ in Pentagon 2 (b) colored zones showing intersections between zone $\mathrm{C}$ in Pentagon 2 and T1, T2 and T3 in Pentagon 1. Zones identified in parenthesis are the ones originally classified in Pentagon 2 (O, C and T3-H).

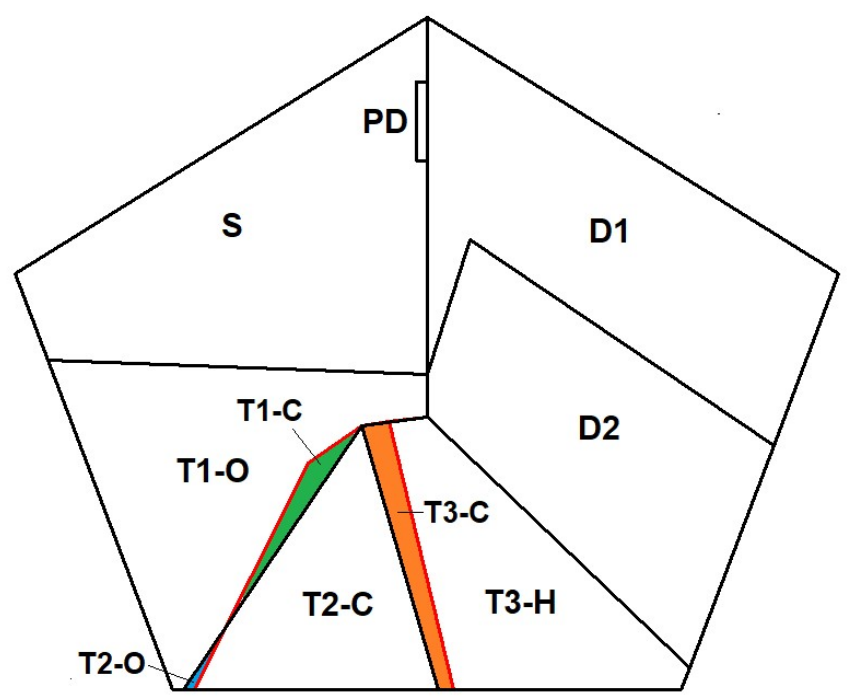

Figure 7. Combined Pentagons 1 and 2, showing colored intersecting zones (in italics below).

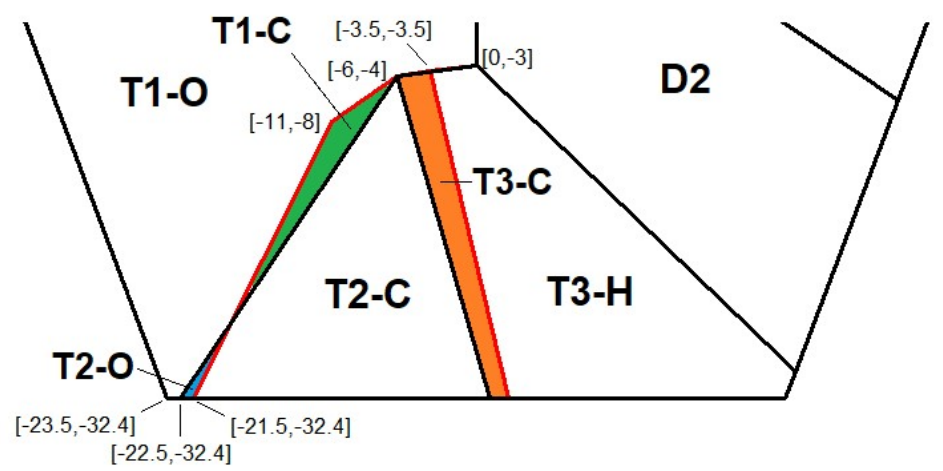

Figure 8. Detailed lower region of the combined Pentagons with the corresponding coordinates. All other zones (PD, S, D1 and D2) remain the same as in the original Pentagons 1 and 2. The orange zone (T3-C) was classified as T3 in Pentagon 1, whereas the green zone (T1-C) was T1 and the blue zone (T2-O) was T2 in Pentagon 1. 


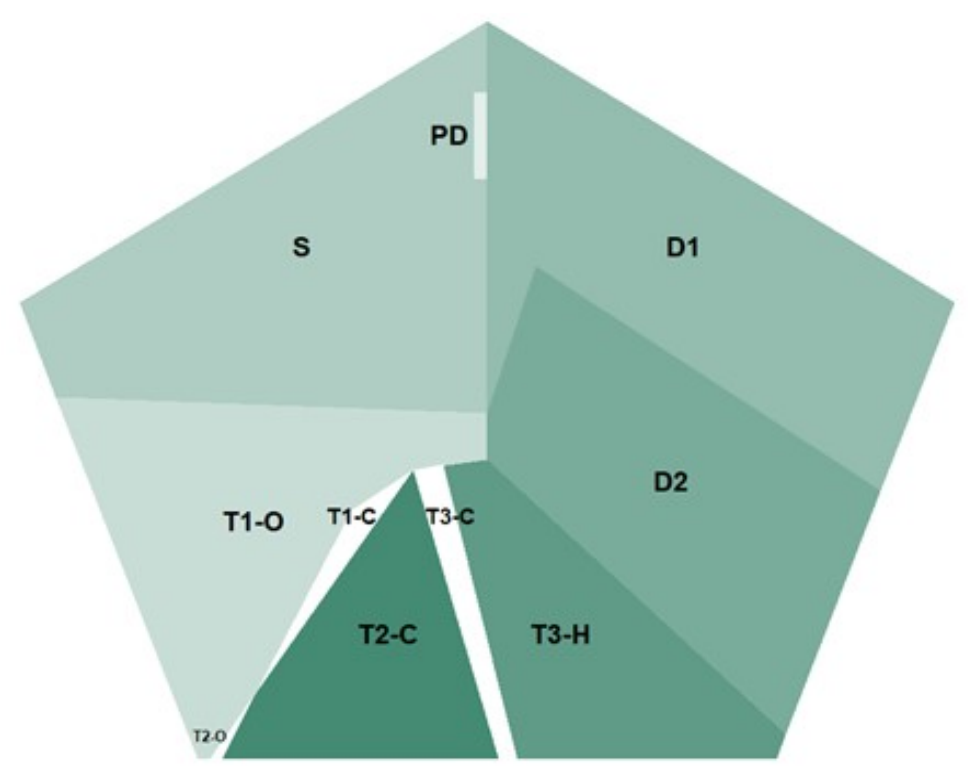

Figure 9. Final geometriy of the newly Combined Pentagons. The 10 zones are PD, S, D1, D2, T1-O, T1-C, T2-O, T2-C, T3-C, T3-H.

T1-O: T1 in Pentagon 1, O in Pentagon 2-confirmation of thermal issue with expected temperature $<300{ }^{\circ} \mathrm{C}$ but without carbonization of solid insulation; T1-C: T1 in Pentagon 1, C in Pentagon 2 -confirmation of thermal issue with expected temperature $<300{ }^{\circ} \mathrm{C}$ but now with likely involvement of paper, showing carbonization; T2-C: T2 in Pentagon 1, C in Pentagon 2; confirmation of thermal issue with temperature in the range 300 to $700{ }^{\circ} \mathrm{C}$, with high likelihood of paper involvement (probability of $80 \%$, based on data from transformers showing faults in internal inspection); T2-O: T2 in Pentagon $1, \mathrm{O}$ in Pentagon 2; confirmation of thermal issue, with a temperature in the range 300 to $700{ }^{\circ} \mathrm{C}$ but unlikely to involve solid insulation or paper carbonization; T3-H: T3 in Pentagon 1, T3-H in Pentagon 2; confirmation of thermal issue in oil only, temperature range above $700{ }^{\circ} \mathrm{C}$; T3-C: T3 in Pentagon $1, \mathrm{C}$ in Pentagon 2; confirmation of high temperature thermal issue (above $700^{\circ} \mathrm{C}$ ) with paper involvement in the fault (carbonization).

An important point to mention is the striking difference between the Duval Method and most of all other methods available in the technical literature which typically are based on gas ratios and in some ranges of those ratios. Most methods will not cover some possible diagnostics if the actual DGA that is being tested with that particular method does not fall within those specific ranges (for example, as in the IEC60599 gas ratio method). The Duval Pentagons will always-and we stress, always—produce a given diagnostic, meaning that if the user runs the method with 100 DGA samples, there will be 100 diagnostics of a given fault zone. The catch here is that one must be very careful on when to use the Pentagons and only apply the technique when there is strong evidence that something is wrong. The method will then provide the fault diagnostic but should never be used to detect a problem. The detection of a problem must precede the diagnostic.

The Combined Pentagon presented in the current article is for those interested in identifying all 10 types of faults, as shown above in Figure 9 (as for instance in IEEE C57.104-2019), with one single geometry, without the need of coding and programming two different Pentagons. It is not intended to replace Pentagons 1 and 2 but to complement them and facilitate the calculation of the 10 types of faults with software applications.

When different diagnoses are given by Pentagons 1, 2, the Combined Pentagon and Triangles 1, 4, 5 for the same DGA analysis, this usually indicates a mixture of faults (multiple faults), which can be identified as described in $[2,6]$ among other publications. 


\section{Discussion of Graphical Accuracy in the Pentagons}

The uncertainty on the position of the DGA point in Duval Triangles, due to the uncertainty on ppm values coming from laboratories, has been calculated in [7] and is represented by an area of uncertainty around the DGA point. A similar calculation was made for the Pentagons, based on a survey [8] carried out by CIGRE involving 25 experienced DGA laboratories in 15 countries. The survey indicated that typical laboratory accuracy, when compared to reference DGA samples, is in the range of $\pm 15 \%$ for samples above minimum detection limits (intermediary gas concentrations). In order to assess the impact of such variability in the DGA measurements, the authors plotted two extreme cases in Pentagon 1, as illustrated in Figure 10 below. The first case, illustrated in Figure 10a, shows the irregular pentagon (in red) formed by the relative contribution of individual gases in a sample where $\mathrm{H}_{2}=300 \mathrm{ppm}, \mathrm{CH}_{4}=600 \mathrm{ppm}, \mathrm{C}_{2} \mathrm{H}_{6}=500 \mathrm{ppm}, \mathrm{C}_{2} \mathrm{H}_{4}=300 \mathrm{ppm}$ and $\mathrm{C}_{2} \mathrm{H}_{2}=30 \mathrm{ppm}$. The red dot shows the centroid of such sample inside Pentagon 1 (the physical location would be the same in any Pentagon, including the combined approach). Notice that we apply a factor of $15 \%$ upwards or downwards to all combustibles in this example, the relative contribution of each gas in the Pentagon would be unchanged and the centroid would also remain the same as in Figure 10a. Put differently, in the extreme cases of a plus or minus error of $15 \%$ to all gases, there would be no difference in the Pentagon classification since the relative contribution of each gas would remain the same. If all gases vary independently in the range $\pm 15 \%$, this results in a possible error in the diagnostic related to the variation of the centroid, as shown in Figure 10b. This variation in the location of the centroid may become more important in cases where the point is near a boundary between two regions, say D1/D2 or T1/T2, etc. The reader must be aware of such possibility and exercise caution when performing DGA with a graphical tool such as the Pentagons.

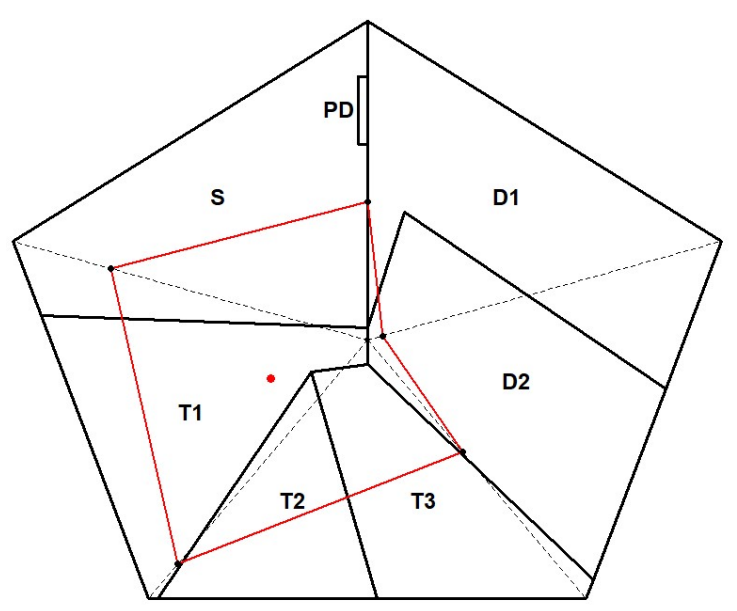

(a)

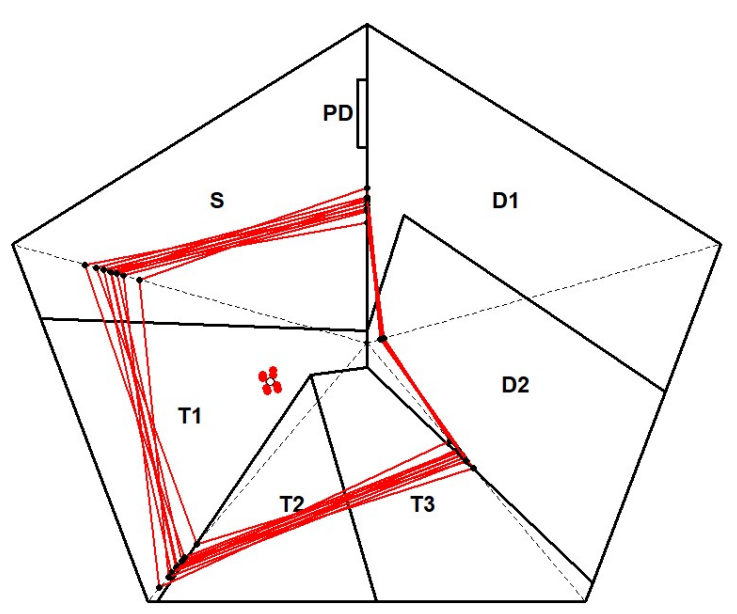

(b)

Figure 10. Illustration of changes in the centroid inside Pentagon 1 , and consequently possible variation in the final DGA diagnostics, given a typical laboratory accuracy of $\pm 15 \%$; (a) when all gases vary simultaneously (b) when each combustible gas varies independently in the range $-15 \%$ to $+15 \%$ relatively to a given central value (assumed to be the reference value).

One must also be aware that such variability in DGA has also an impact in other methods that utilize gas ratios, such as the IEC 60599 method. The cases discussed below will only consider the central value of the DGA samples as those given in the cited reference and that did not mention their laboratory accuracies.

\section{Examples of Real DGA Cases Classified in all Pentagons}

In order to verify the application of the Combined Pentagons with real cases of Dissolved Gas Analysis from actual transformers that were inspected internally to confirm diagnostics, we utilized a 
few examples taken from a recent CIGRE document [9], that summarizes the work of a joint working group between the Study Committee D1 (Materials and Emerging Test Techniques) and the Study Committee A2 (Transformers), forming the JWG D1/A2.47 Advances in DGA Interpretation.

The document presents a significant number of cases that involve faults of several types but only a few cases involving thermal issues were selected to illustrate the region of interest in all Pentagons for the current proposal. All cases illustrate faulty transformers that were suspicious and therefore thoroughly investigated even by internal inspection.

A brief description of the selected cases is given in Table 1 and Figure 11 below. Notice that the summary of the problem found during internal inspection is indicated in Table 1, together with the page from which each case was taken from that reference CIGRE document. Figure 11 shows the diagnostic found by the utilization of both Pentagon 1 and 2, and the Combined Pentagons.

Table 1. Illustration of five DGA cases given in [9] with internal inspection to confirm diagnostics.

\begin{tabular}{|c|c|c|c|c|c|c|c|c|c|c|c|c|}
\hline Case & Ref. [9] Page & $\mathbf{H}_{2}$ & $\mathrm{CH}_{4}$ & $\mathrm{C}_{2} \mathrm{H}_{2}$ & $\mathrm{C}_{2} \mathrm{H}_{4}$ & $\mathrm{C}_{2} \mathrm{H}_{6}$ & $\mathrm{CO}$ & $\mathrm{CO}_{2}$ & \multicolumn{3}{|c|}{ Pentagon } & Internal Inspections \\
\hline 1 & 20 & 29 & 204 & 0 & 17 & 264 & 24 & 2000 & $\mathrm{~T} 1$ & $\mathrm{O}$ & $\mathrm{T} 1-\mathrm{O}$ & Overheated iron sheets \\
\hline 3 & 24 & 754 & 2647 & 6 & 2590 & 1127 & 105 & 538 & $\mathrm{~T} 2$ & $\mathrm{C}$ & $\mathrm{T} 2-\mathrm{C}$ & Carbonized leads \\
\hline 4 & 27 & 2070 & 31879 & 55 & 38192 & 1127 & 324 & 3000 & T3 & $\mathrm{C}$ & T3-C & Carbonized paper \\
\hline
\end{tabular}

Case 1: Faults classified as type $\mathrm{O}$ (overheating)—no indication of paper involvement

This is one of several cases reported in the CIGRE document and to the JWG D1/A2.47 in which the fault is typically dominated by ethane $\left(\mathrm{C}_{2} \mathrm{H}_{6}\right)$ in the range $400-1200 \mathrm{ppm}$. As it can be seen in Table 1 and in Figure 11, this specific case was classified as a thermal issue of temperature $\mathrm{T}<300{ }^{\circ} \mathrm{C}$ in Pentagon 1 (Figure 10a), classified as overheating $(\mathrm{O})$ in Pentagon 2 (Figure 11b) and consequently classified as T1-O (Figure 11c) in the Combined Pentagons.

Case 2: Faults of very high temperature in oil only (T3-H)

These are faults of high temperature typically dominated by ethylene $\left(\mathrm{C}_{2} \mathrm{H}_{4}\right)$ in the range 120-450 ppm, as reported by the CIGRE JWG. This type of fault, although of very high temperature, in general, does not lead to a full-blown transformer failure since it occurs in the oil only, hence the active parts (core and windings) are not involved. For this specific case, it was classified as thermal issue of very high temperature (T3) in Pentagon 1, as high temperature issue in oil only (T3-H) in Pentagon 2 and, consequently, classified as T3-H in the Combined Pentagons.

Case 3: Carbonization of solid insulation with intermediary temperature

Ethylene $\left(\mathrm{C}_{2} \mathrm{H}_{4}\right)$ tends to be the dominating gas in this type of fault as well, with a typical range reported by the JWG 200-700 ppm, showing temperatures from 300 to $700{ }^{\circ} \mathrm{C}$. As can be seen in Figure 11a, this case was classified as T2 in Pentagon 1, C in Pentagon 2 (Figure 11b) and T2-C in the Combined Pentagons (Figure 11c).

Case 4: Carbonization of solid insulation between winding turns, high temperature

The CIGRE JWG reports a typical range of ethylene $\left(\mathrm{C}_{2} \mathrm{H}_{4}\right) 2-7 \mathrm{ppm}$, much lower than in previous cases. One additional possibility of fault identification of this kind is to follow changes from $\mathrm{O}$ (overheating) into the $\mathrm{C}$ zone of the Pentagons. This specific case was classified as T3 in Pentagon 1, Figure 11a, C in Pentagon 2 (borderline in Figure 11b), and consequently T3-C in the Combined Pentagons. Note that this case fell on the border between zones $\mathrm{C}$ and T3-H in Pentagon 2, indicating that it could go either way. This is not uncommon and can be explained by factors such as inaccuracies in the measurement of dissolved gases in oil which can lead to variations in the calculated centroid inside each Pentagon, as explained in session 6 above, sample contamination, laboratory error, etc., in addition to the very nature of the Pentagon method that establishes a physical border between zones. It is, therefore, expected that some DGA results, when plotted on the Pentagons, will fall on 
those borderlines. A possible approach is to always choose the most critical diagnostic or the diagnostic that can lead to the most costly and damaging result. Since in this case the choice would be between involving solid insulation ( $\mathrm{C}$ for carbonization) or not involving solid insulation (fault $\mathrm{T} 3-\mathrm{H}$ in oil only), the decision is clear if one wants to operate on the safe side. A problem involving solid insulation may lead the transform to a catastrophic failure, whereas faults involving oil only would hardly lead to that extent.

Case 5: Localized carbonization of solid insulation between winding turns

This case is a very good example of carbonization of paper insulation at moderate temperatures, typical $\mathrm{T}<300^{\circ} \mathrm{C}$. As illustrated in Figure 11a, it was classified as T1 in Pentagon 1, as C in Pentagon 2, and consequently T1-C in the Combined Pentagons.

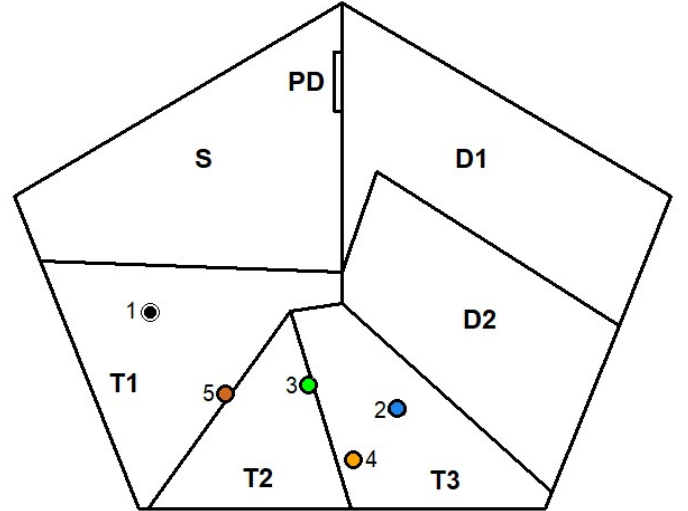

(a)

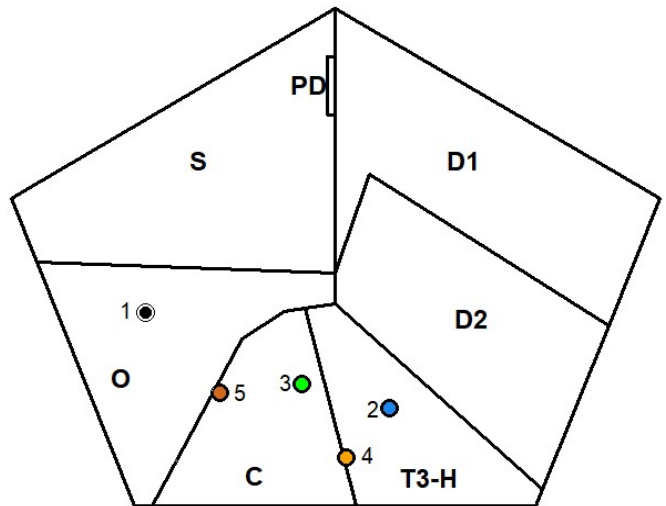

(b)

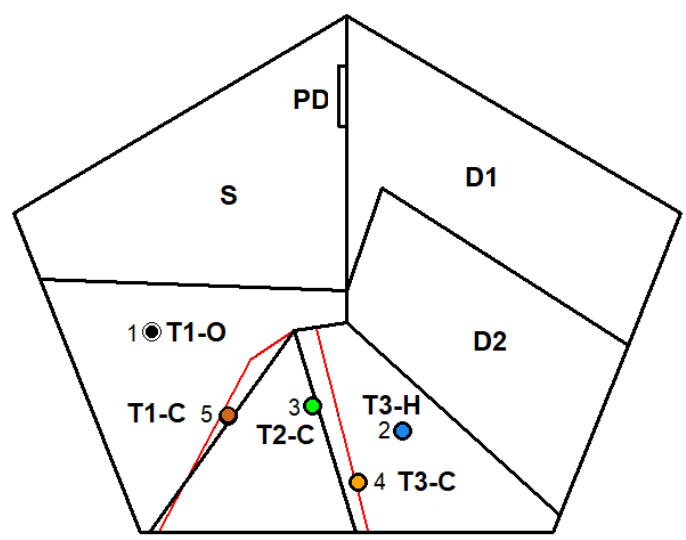

(c)

Figure 11. Illustration of five DGA cases shown in Table 1 for which there was internal inspection of the transformers in order to identify any developing issue; (a) classification of all cases in Pentagon 1 (b) classification of all cases in Pentagon 2; (c) classification of all cases in the Combined Pentagons.

\section{Conclusions}

Users not familiar with the use of the Pentagon method may be puzzled by the fact that we now apparently have three Pentagons instead of two. This is not correct. Pentagon 1 is intended for DGA users and transformer experts that are familiar only with the six basic types of faults (PD, D1, D2, T1, T2 and T3) used in IEC 60599 [1] and the Duval Triangle 1 method, also given in the IEC60599.

Pentagon 2 is for those familiar with the four additional types of faults which have been used in Duval Triangles 4 and 5 since 2008 (zones S, O, C and T3-H), facilitating actions on the equipment when such faults can be identified.

The Combined Pentagons proposed in this present article encompasses all the above since it classifies the main types of fault and also provides resolution into the several sub types, as in $\mathrm{S}, \mathrm{O}, \mathrm{C}$, 
etc. The work carried out in this paper demonstrates that the Combined Pentagons does not change the expected diagnostics of Pentagons 1 and 2 and certainly facilitates computational calculations, since it utilizes a single geometry to represent both Pentagons.

It is also very important to point out that, in all cases illustrated in Table 1, the diagnostics obtained by the Pentagons method agreed $100 \%$ with the findings of the internal inspections that were carried out by the experts that provided the data to the CIGRE JWG D1/A2.47 [9].

Author Contributions: Conceptualization, L.C.; Methodology, L.C. and M.D.; Software and Validation, L.C. and S.H.; Formal Analysis, M.D. and L.C.; Writing-Original Draft Preparation, L.C., S.H. and M.D.; Writing-Review \& Editing, L.C., S.H. and M.D.; Visualization, L.C.; Supervision, L.C. and M.D. All authors have read and agreed to the published version of the manuscript.

Funding: This research received no external funding and was thoroughly supported by the affiliated companies of all authors.

Acknowledgments: The authors would like to acknowledge both ABB Power Grids and IREQ for supporting this research.

Conflicts of Interest: The authors declare no conflict of interest.

\section{References}

1. IEC. IEC 60599-Mineral Oil-Impregnated Electrical Equipment in Service - Guide to the Interpretation of Dissolved and Free Gases Analysis; International Electrotechnical Commission: Geneva, Switzerland, 2015.

2. IEEE. IEEE C57.104-2019-Guide for the Interpretation of Gases Generated in Mineral Oil-Immersed Transformers; Institute of Electrical and Electronics Engineers: Piscataway, NJ, USA, 2019.

3. Cheim, L.; Platts, D.; Prevost, T.; Xu, S. Furan Analysis for Liquid Power Transformers. IEEE Electr. Insul. Mag. 2012, 28, 8-21. [CrossRef]

4. CIGRE. CIGRE Brochure 494—Furanic Compounds for Diagnosis; CIGRE: Paris, France, 2012.

5. Duval, M.; Lamarre, L. The Duval Pentagon-A new complementary tool for the interpretation of dissolved gas analysis in Transformers. IEEE Electr. Insul. Mag. 2014, 30, 9-12.

6. Duval, M. DGA Tutorial. In Proceedings of the TECHCON Conference, Albuquerque, NM, USA, 11-13 September 2016.

7. Duval, M.; Dukarm, J. Improving the reliability of transformers gas-in-oil analysis. IEEE Electr. Insul. Mag. 2005, 21, 21-27. [CrossRef]

8. Duval, M. New techniques for dissolved gas in oil analysis. IEEE Electr. Insul. Mag. 2003, 19, 6-15. [CrossRef]

9. CIGRE. CIGRE Brochure 771-Advances in DGA interpretation; CIGRE: Paris, France, 2019; pp. 20-29. 